\title{
BMJ Open Long-term non-invasive ventilation therapies in children: a scoping review protocol
}

\author{
Maria L Castro Codesal, ${ }^{1,2}$ Robin Featherstone, ${ }^{1,3}$ Carmen Martinez Carrasco, ${ }^{4}$ \\ Sherri L Katz, ${ }^{5}$ Elaine Y Chan, ${ }^{6}$ Glenda N Bendiak, ${ }^{7}$ Fernanda R Almeida, ${ }^{8}$ \\ Rochelle Young, ${ }^{2}$ Deborah Olmstead, ${ }^{2}$ Karen A Waters, ${ }^{9}$ Collin Sullivan, ${ }^{9}$ \\ Vicki Woolf, ${ }^{10}$ Lisa Hartling, ${ }^{1,3}$ Joanna E MacLean ${ }^{1,2}$
}

To cite: Castro Codesal ML, Featherstone R, Martinez Carrasco C, et al. Long-term non-invasive ventilation therapies in children: a scoping review protocol. BMJ Open 2015;5:e008697. doi:10.1136/bmjopen-2015008697

- Prepublication history for this paper is available online. To view these files please visit the journal online (http://dx.doi.org/10.1136/ bmjopen-2015-008697).

Received 15 May 2015 Revised 15 July 2015 Accepted 23 July 2015

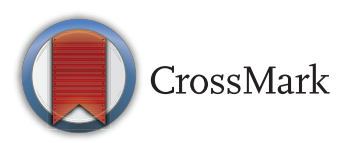

For numbered affiliations see end of article.

Correspondence to Dr Maria L Castro Codesal; castroco@ualberta.ca

\begin{abstract}
Introduction: Non-invasive ventilation (NIV) in children has become an increasingly common modality of breathing support where pressure support is delivered through a mask interface or less commonly through other non-invasive interfaces. At this time, NIV is considered a first-line option for ventilatory support of chronic respiratory insufficiency associated with a range of respiratory and sleep disorders. Previous reviews on the effectiveness, complications and adherence to NIV treatment have lacked systematic methods. The purpose of this scoping review is to provide an overview of the evidence for the use of long-term NIV in children.
\end{abstract}

Methods and analysis: We will use previously established scoping methodology. Ten electronic databases will be searched to identify studies in children using NIV for longer than 3 months outside an intensive care setting. Grey literature search will include conference proceedings, thesis and dissertations, unpublished trials, reports from regulatory agencies and manufacturers. Two reviewers will independently screen titles and abstracts for inclusion, followed by full-text screening of potentially relevant articles to determine final inclusion. Data synthesis will be performed at three levels: (1) an analysis of the number, publication type, publication year, and country of publication of the studies; (2) a summary of the study designs, outcomes measures used; (3) a thematic analysis of included studies by subgroups.

Ethics and dissemination: This study will provide a wide and rigorous overview of the evidence on the use of long-term NIV in children and provide critical information for healthcare professionals and policymakers to better care for this group of children. We will disseminate our findings through conference proceedings and publications, and evaluate the results for further systematic reviews and meta-analyses.

\section{BACKGROUND}

The purpose of this scoping review is to provide a rigorous overview of the evidence

\section{Strengths and limitations of this study}

- This is a novel review approach to define the full scope of literature available on the use of longterm non-invasive ventilation in children.

- In addition to methodological experts, key knowledge translation partners were engaged to support the design of the protocol.

- Subgroup for separate summary will be determined after review of the identified literature.

- As this is a scoping review, the quality of the evidence and risk of bias will not be evaluated.

- This review will be limited to English, French, Spanish, Portuguese, Italian and Catalan.

for the use of long-term non-invasive ventilation (NIV) in children with respiratory and/ or sleep disorders. NIV in children is an increasingly common modality of breathing support where a mask interface, rather than an endotracheal tube or tracheostomy, is used to connect to pressure support. Long-term use of NIV provides an option for children who require intermittent ventilatory support (ie, for sleep only) or as part of palliative care. The use of NIV in children has expanded worldwide including developing countries, since its first reported use in the early 1990s, resulting in a decrease in the use of long-term invasive ventilation via tracheostomy. ${ }^{1-11}$ Factors contributing to the increased use of NIV likely include increased survival of children with complex medical conditions, a shift in the emphasis of healthcare from hospital to home based care, and technological advances in the masks and machines to support home use of NIV in children.

Today, NIV is considered a first-line option of ventilatory support for chronic respiratory insufficiency associated with a range of respiratory and sleep disorders including 
chronic respiratory failure ${ }^{12-14}$ cystic fibrosis,${ }^{15-18}$ musculoskeletal weakness or chest wall restriction, ${ }^{19-24}$ obstructive sleep apnoea (OSA) and craniofacial malformations, ${ }^{25-28}$ sleep disorders associated with neurological conditions and abnormalities in central respiratory drive. ${ }^{29-32}$ Long-term NIV has been used successfully in infants, from the first weeks of life. It has been shown NIV can be used long-term across all age groups. ${ }^{33-37}$ Safety and efficacy of NIV use in children have been documented for children with certain underlying conditions such as neuromuscular disease or OSA. ${ }^{38-40}$ Adherence to NIV therapy is also an outcome of interest as there are considerable challenges in establishing and maintaining use of NIV in children. ${ }^{41-46}$ Reported benefits of NIV use in children are broad and include outcomes related to acute illness, ${ }^{47}$ chronic respiratory function, ${ }^{18} 2348$ sleep and daytime function, ${ }^{28} 49{ }^{50}$ behaviour and neurocognitive outcomes, ${ }^{51}$ general health outcomes, ${ }^{20}$ quality of life, ${ }^{49}{ }^{52}$ progression of underlying disease and survival. ${ }^{52}{ }^{53}$ Funding for NIV and access to this technology are also important considerations. ${ }^{54}$

Previous reviews on long-term NIV use in children have lacked systematic methodology or NIV in children has been included in systematic reviews of broader topics. From a preliminary search strategy using terms related to NIV and children, we identified 758 review articles of which 91 may have some information relevant to longterm NIV use in children. Of these, only eight previous reviews described a search strategy or systematic methodology. ${ }^{32}{ }^{55-61}$ Of note, none of the reviews had a specific focus of enquiry regarding long-term use of NIV in children but rather included NIV as one possible intervention for a particular paediatric population. Moreover, only four of these reviews had a specific section on NIV or included studies with NIV interventions. The aim of this scoping review is to define the body of literature relevant for the use of long-term NIV in children and provide a systematic overview of the existing evidence. Using this process, we will not only define the scope of existing data but also determine if there is sufficient literature relevant to subgroups that would be appropriate to apply systematic review or meta-analysis methodology.

\section{METHODS AND ANALYSIS}

\section{Study design}

The methodology of this scoping review is based on the methodological frameworks developed by Bragge et $a b^{62}$ and Arksey and O'Malley. ${ }^{63}$ The results will be reported following the "Preferred Reporting Items for Systematic Reviews and Meta-Analyses protocols (PRISMA-P) 2015 statement". ${ }^{64}$ This approach will enable a rational assessment of the evidence that is available on long-term NIV interventions in children and ensure a transparent and complete report of the same.

We gathered an advisory team of experts in systematic reviews, pediatric respirologists, sleep medicine specialists, paediatric nurse practitioners and industry representatives, to provide input on the search strategy as well as in the discussion of the results of the scoping review. In addition, children using long-term NIV and their caregivers have been included in the advisory team with the aim of prioritising the outcomes identified in this scoping review from the patient and caregiver perspective as well as identifying important outcomes that have not been included in previous studies.

\section{Eligibility criteria}

Types of participants/population: Studies reporting results for children from newborn to age 18 years will be included. Studies that include adults and children will be included if data for children is reported separately.

Types of Interventions: Eligible studies must contain information on NIV use defined as the administration of breathing support delivered through a non-invasive interface most commonly a nasal or face mask but also a mouth piece or abdominal belt. Types of breathing support includes (1) positive pressure support including continuous positive airway pressure (CPAP) or an inspiratory and expiratory airway pressure (bi-level), (2) negative pressure ventilation (NPV). Similar to the definition we found in other review articles from the preliminary search review, we will define long-term use as the use of NIV for at least 3 months outside an acute care environment. This may include, but not be limited to, use of NIV in community-based settings such as homes, a family or group home or specialised non-acute hospital based units. This definition is intended to include children with chronic conditions that require long-term NIV and are stable enough to receive ventilatory therapy at home or in a chronic care environment. Articles that describe longterm use of NIV with no time specification will be included if there is a clear intention of treating a chronic problem.

Type of outcomes: No restriction with regard to outcomes will be applied.

Types of Studies: Original studies published from 1990 onwards will be considered for inclusion including randomised and non-randomised clinical trials, controlled before-after studies, cross-sectional studies, longitudinal observational studies, retrospective cohorts, qualitative and mixed methods research and case series with three or more cases; 1990 was determined as a starting date because the first study of long-term NIV use in children we identified was published in 1993.

Exclusion: Case reports, case series with less than three subjects, comments, editorials, letters and reviews will be excluded. Studies where NIV is used solely for the treatment of acute illness will be excluded. During the fulltext screening, only articles in English, French, Spanish, Portuguese, Italian and Catalan will be included as the review authors are proficient in those languages.

Information sources: An information specialist working for the Alberta Research Centre for Health Evidence (ARCHE) at the University of Alberta collaborated with investigators to design a comprehensive and sensitive 
Box 1 Search strategy developed for MEDLINE using OVID

\section{Search terms}

1. Continuous Positive Airway Pressure/

2. Noninvasive Ventilation/

3. Intermittent Positive-Pressure Breathing/

4. Ventilators, Negative-Pressure/

5. AVAPS.tw.

6. ((auto* or adaptive) adj2 (servoventilation or ventilation)).tw.

7. AutoSet ${ }^{\star}$. tw.

8. ((bi level or bilevel) adj2 (airway* or air way* or assist* ${ }^{*}$ or breath $^{*}$ or positive pressure* ${ }^{\star}$ or respirat ${ }^{\star}$ or ventilat ${ }^{*}$ or support* $^{*}$ or therap $\left.{ }^{\star}\right)$ ).tw.

9. BIPAP* $^{*} . \mathrm{tw}$.

10. $B P A P^{*} . t w$.

11. c flex.tw.

12. CNEP.tw.

13. (continuous negative adj2 pressure).tw.

14. (continuous positive airway* or continuous positive air way $\left.{ }^{\star}\right)$.tw.

15. (continuous positive adj2 pressure).tw.

16. CPAP $^{*}$.tw.

17. ((domicil ${ }^{\star}$ or home $\left.{ }^{\star}\right)$ adj5 ventilat $\left.{ }^{\star}\right)$.tw.

18. intermittent positive pressure breathing.tw.

19. $\mathrm{IPPB}^{*}$.tw.

20. ((long term or longterm) adj5 ventilat $\left.{ }^{\star}\right)$. tw.

21. ((nasal ${ }^{*}$ or mask $\left.{ }^{*}\right)$ adj2 (positive adj2 pressure)).tw.

22. ((nasal ${ }^{\star}$ or mask $\left.^{\star}\right)$ adj2 ventilat $\left.{ }^{\star}\right)$.tw.

23. nCPAP* $^{*}$.tw.

24. ((negative pressure) adj2 (respirat* or ventilat $\left.\left.{ }^{\star}\right)\right) . t w$.

25. ((night ${ }^{\star}$ or nocturnal ${ }^{\star}$ or sleep $\left.{ }^{\star}\right)$ adj5 ventilat $\left.{ }^{\star}\right)$.tw.

26. NIPPV*.tw.

27. ((noninvasive adj5 ventilat $\left.{ }^{\star}\right)$ or (non invasive adj5 ventilat $\left.{ }^{\star}\right)$ ). tw.

28. (noninvasive respiratory support ${ }^{\star}$ or non invasive respiratory support $\left.^{\star}\right)$.tw.

29. NPPV*.tw.

30. (positive pressure adj2 respirat $\left.^{\star}\right)$.tw.

31. REMstar*.tw.

32. (tank adj (respirat ${ }^{\star}$ or ventilat $\left.\left.{ }^{\star}\right)\right) . t w$.

33. VPAP*.tw.

34. $\mathrm{or} / 1-33$

35. Hypoventilation/pc, rh, th [Prevention \& Control, Rehabilitation, Therapy]

36. Interactive Ventilatory Support/

37. Intermittent Positive-Pressure Ventilation/

38. Positive-Pressure Respiration/

39. Respiration, Artificial/

40. Respiratory Insufficiency/pc, rh, th [Prevention \& Control, Rehabilitation, Therapy]

41. exp Sleep Apnea Syndromes/ pc, rh, th [Prevention \& Control, Rehabilitation, Therapy]

42. Ventilators, Mechanical/

43. ((airway ${ }^{\star}$ or air way* or breath* ${ }^{\star}$ or inspirat ${ }^{\star}$ or respirat ${ }^{\star}$ or ventilat $\left.^{\star}\right)$ and (positive adj2 pressure)).tw.

44. intermittent positive pressure.tw.

45. IPPV ${ }^{*}$.tw.

46. (mechanical adj (respirat ${ }^{\star}$ or ventilat $\left.\left.^{\star}\right)\right)$.tw.

47. (positive adj2 pressure adj (assist* or support* ${ }^{\star}$ or therap $\left.{ }^{\star}\right)$ ). tw.
Box 1 Continued

48. positive airway pressure.tw.

49. pulmonary ventilator*.tw.

50. respiratory support ${ }^{\star}$. tw.

51. or $/ 35-50$

52. (noninvasive or non invasive or spontaneous*).mp.

53. 51 and 52

54. 34 or 53

55. $\exp$ Adolescent/

56. $\exp$ Child/

57. $\exp$ Infant/

58. exp Minors/

59. $\exp$ Pediatrics/

60. $\exp$ Puberty/

61. $\exp$ Schools/

62. adoles*.mp.

63. (baby ${ }^{*}$ or babies or infant* ${ }^{*}$ or infancy or neonat ${ }^{*}$ or newborn ${ }^{\star}$ or postmatur ${ }^{\star}$ or prematur* or preterm $\left.{ }^{\star}\right) . m p$.

64. (boy $^{\star}$ or girl ${ }^{\star}$ or teen*).mp.

65. (child* or kid or kids or preschool* or school age* or schoolchild $^{\star}$ or toddler $\left.{ }^{\star}\right)$.mp.

66. (elementary school ${ }^{*}$ or high school $^{*}$ or highschool ${ }^{*}$ or kindergar $^{\star}$ or nursery school ${ }^{\star}$ or primary school ${ }^{\star}$ or secondary school $\left.^{\star}\right) \cdot \mathrm{mp}$.

67. minors*.mp.

68. (paediatric ${ }^{\star}$ or peadiatric ${ }^{\star}$ or pediatric ${ }^{\star}$ ).mp.

69. (prepubescen ${ }^{\star}$ or pubescen ${ }^{\star}$ or pubert $\left.{ }^{\star}\right) \cdot m p$.

70. or $/ 55-69$

71. 54 and 70

72. (case reports or comment or editorial or letter).pt.

73. 71 not 72

74. exp animals/not humans.sh.

75. 73 not 74

76. limit 75 to $y r=" 1990$-Current"

77. remove duplicates from 76

search strategy with terms for NIV and children from newborn to age 18 years. The search strategy (box 1) was developed for Ovid Medline with a validated child search filter, ${ }^{65}$ and will be translated into Ovid Embase, PubMed (last year only), CINAHL via EbscoHOST, and Wiley Cochrane Library (including the Cochrane Database of Systematic Reviews, the Cochrane Central Register of Controlled Trials, the Database of Abstracts of Reviews of Effects, the Health Technology Assessment Database, and the NHS Economic Evaluation Database). Database search results will be limited to human studies published after 1990. No language or study design restrictions will be applied.

Reference lists of all studies selected for this scoping review will be scanned to identify further relevant studies not detected by the search strategy.

We will also search for grey literature (non-peer reviewed investigations) including conference proceedings, thesis and dissertations, unpublished trials, regulatory agencies and manufacturers reports. In consultation with the advisory team, a list of conference proceedings and annual meeting reports to review from January 2012 
to December 2014 will be established. The information specialist will search websites of the conferences relevant to NIV such as the American Thoracic Society, the American College of Chest Physicians, the Canadian Thoracic Society, the European Respiratory Society, the American Academy of Sleep Medicine, the European Society of Sleep Research, the Australasian Sleep Association and the American Association of Neuromuscular and Electrodiagnostic Medicine. Investigators will search for publications of relevant proceeding abstracts or contact presenters to request study data if needed. ClinicalTrials.gov and the World Health Organisation's International Clinical Trials Registry Platform will also be searched for trials registered after 2012 on NIV and positive airway pressure. Investigators will search for publications of relevant trials or attempt to contact study coordinators to request unpublished data. Regulatory agencies will be searched for ventilator device approval documents, premarket notifications, recall notices and safety advisories. Government sources will include Health Canada's Medical Device Active License Listing (MDALL), the U.S. Food and Drug Administration (FDA) device website (Devices@FDA), the Australian Government's Department of Health and Ageing Therapeutic Goods Administration (TGA) Database of Adverse Event Notifications, the European Medicines Agencies, and the New Zealand Medicines and Medical Devices Safety Authority (Medsafe: Medical Devices). Device manufacturers may be contacted with requests for premarket trial data. ProQuest Theses \& Dissertations will also be searched for theses submitted after 1990 on NIV in children.

The Ovid Medline search strategy and the list of information sources will be approved by the advisory team prior to running the searches.

\section{Study records}

Data management: Results of searches will be imported into an EndNote library, and duplicates will be removed. Two exact copies of the EndNote library will be created for independent screening by two reviewers.

Selection process: Two independent reviewers will screen titles and abstracts of retrieved articles for eligibility based on the inclusion criteria. The full text will be retrieved for all potentially relevant articles; each will be evaluated independently for eligibility by two reviewers. Discrepancies will be resolved through discussion between the reviewers to establish the final list of studies to be included in the scoping review. Reasons for exclusion will be recorded at the full-text review.

Data collection process: Data extraction will be completed by one reviewer using a pre-designed standardised form and entered into Microsoft Excel database (Microsoft, Redmond, Washington, USA). Data extraction will be

Table 1 Data extraction form

\begin{tabular}{|c|c|}
\hline Study characteristics & Extracted data \\
\hline \multirow[t]{6}{*}{ General information } & First author last name \\
\hline & Title \\
\hline & Journal \\
\hline & Publication year \\
\hline & Country of study/continent/multinational \\
\hline & Publication type: journal, abstract, dissertation, unpublished trial, report \\
\hline \multirow[t]{3}{*}{ Introduction } & Aims of the study \\
\hline & Study research question \\
\hline & $\begin{array}{l}\text { Study population: number of subjects using NIV, mean age, age range, gender, } \\
\text { primary underlying condition, comorbidities }\end{array}$ \\
\hline \multirow[t]{6}{*}{ Design } & Study design: \\
\hline & $\begin{array}{l}\text { Quantitative: randomised controlled trial, non-randomised controlled trial, } \\
\text { controlled before-after studies, observational, cross-sectional } \\
\text { Qualitative: case series, ethnography, grounded theory, phenomenology, } \\
\text { other, mixed methods }\end{array}$ \\
\hline & Sample size \\
\hline & $\begin{array}{l}\text { Intervention type, NIV term used, interface type, duration of intervention, } \\
\text { cointerventions }\end{array}$ \\
\hline & Statistical analysis methods used \\
\hline & Control group: number of control subjects, $y / n$, intervention in control group \\
\hline \multirow{4}{*}{$\begin{array}{l}\text { Outcomes measures (whether self-reported } \\
\text { or objective tools)* }\end{array}$} & Primary outcomes \\
\hline & Secondary outcomes \\
\hline & Adverse outcomes \\
\hline & Duration of the follow-up \\
\hline Authors conclusions & Positive, negative, neutral, indeterminate \\
\hline Gaps and limitations identified by authors & \\
\hline
\end{tabular}


verified by a second reviewer for a sample of $10 \%$ of the studies. The data extraction form is based on Bragge et $a \bar{\nu}^{6}$ data extraction database and modified for this project to ensure that appropriate and relevant data is obtained (table 1). When there is missing information, two attempts to contact the corresponding authors will be made to obtain additional data. To avoid double counting in the instance of the same data set published in more than one publication, only one article per data set will be retained.

Data synthesis: The identified evidence will be collated using a specific analytical framework in order to present a narrative account of the existing literature.

Once the information has been extracted, we will present a narrative account of findings in three different ways: (1) a basic numerical analysis of the number, publication type, publication year and country of publication of the studies included in the review. (2) A narrative description of the study design, aims, participant characteristics, sample size, intervention type, control group description, outcomes measures, and statistical methods. We will use this information to establish subcategorise of studies which may include grouping based on age (eg, infants, children, and adolescents), intervention type (eg, CPAP, bi-level, and NPV) and disease categories (eg, OSA, neuromuscular disease). (3) A thematic analysis of included studies by subgroups if appropriate. The results will be presented based on the priorities established by our advisory team including input from children using NIV and their caregivers.

Timeline: We anticipate finishing the search, screening, data extraction and synthesis within 6 months. A search update may be required if the timeline is longer than expected.

\section{CONCLUSION}

This scoping review will be the first, to our knowledge, to provide a systematic overview of the evidence on the use of long-term NIV in children. The findings from this review will provide stakeholders with a rigorous research base to support healthcare providers to improve clinical practice and policymakers to support resource needs for this complex group of children. We will disseminate our findings through conference proceedings and publications. The gathered data can be used to inform the development of guidelines for the care of children using long-term NIV and will identify gaps in knowledge to support future research endeavours. Based on the results, we will determine whether the application of other systematic review methodologies, such as meta-analysis or meta-synthesis, will be appropriate for any of the subgroups that we identify for future research.

\footnotetext{
Author affiliations

${ }^{1}$ Department of Pediatrics, University of Alberta, Edmonton, Alberta, Canada

${ }^{2}$ Stollery Children's Hospital, Edmonton, Alberta, Canada

${ }^{3}$ Alberta Research Centre for Health Evidence, University of Alberta,

Edmonton, Alberta, Canada
}

${ }^{4}$ Department of Pediatrics, Hospital La Paz, Madrid, Spain

${ }^{5}$ Department of Pediatrics, University of Ottawa and Children's Hospital of Eastern Ontario, Ottawa, Ontario, Canada

${ }^{6}$ Department of Respiratory Medicine, Great Ormond Street Hospital for Children, London, UK

${ }^{7}$ Department of Pediatrics, University of Calgary, Calgary, Alberta, Canada

${ }^{8}$ Faculty of Dentistry, University of British Columbia, Vancouver, British Columbia, Canada

${ }^{9}$ Sydney Medical School, University of Sydney, Sydney, New South Wales, Australia

${ }^{10}$ Sleep Medix Inc, Edmonton, Alberta, Canada

Contributors MLCC, RF, LH, and JEM conceived the idea of this study and designed its methodology. MLCC and RF wrote the protocol, developed the search strategy and performed a preliminary literature review. KAW, CMC, SLK, EYC, GNB, CS, FRA, RY, DO, VW, LH and JEM reviewed the protocol. All authors read and approved the final manuscript.

Funding This work has been funded by the Women and Children's Health Research Institute (WCHRI) through the generous support of the Stollery Children's Hospital Foundation. MCC received salary support through a Stollery Clinical Research Fellowship. The funders had no role in the study design, data collection and analysis, decision to publish, or preparation of the manuscripts. Dr Lisa Hartling is supported by a CIHR New Investigator Salary Award.

Competing interests None declared.

Provenance and peer review Not commissioned; externally peer reviewed.

Data sharing statement The protocol of this scoping review, the reference list of included articles, additional unpublished data from the preliminary search strategy and a summary of previous review articles on NIV in children are available by contacting the corresponding author.

Open Access This is an Open Access article distributed in accordance with the Creative Commons Attribution Non Commercial (CC BY-NC 4.0) license, which permits others to distribute, remix, adapt, build upon this work noncommercially, and license their derivative works on different terms, provided the original work is properly cited and the use is non-commercial. See: http:// creativecommons.org/licenses/by-nc/4.0/

\section{REFERENCES}

1. Kamm M, Burger R, Rimenzsberger $P$, et al. Survey of children supported by long-term mechanical ventilation in Switzerland. Swiss Med Wkly 2001;131:261-6.

2. Fauroux B, Boffa C, Desguerre I, et al. Long-term noninvasive mechanical ventilation for children at home: a national survey. Pediatr Pulmonol 2003;35:119-25.

3. Edwards EA, Hsiao K, Nixon GM. Paediatric home ventilatory support: the Auckland experience. J Paediatr Child Health 2005;41:652-8.

4. Gowans M, Keenan HT, Bratton SL. The population prevalence of children receiving invasive home ventilation in Utah. Pediatr Pulmonol 2007;42:231-6.

5. Graham RJ, Fleegler EW, Robinson WM. Chronic ventilator need in the community: a 2005 pediatric census of Massachusetts. Pediatrics 2007;119:e1280-e87.

6. Racca F, Bonati M, Del Sorbo L, et al. Invasive and non-invasive long-term mechanical ventilation in Italian children. Minerva Anestesiol 2011;77:892-901.

7. Wallis $C$, Paton JY, Beaton $S$, et al. Children on long-term ventilatory support: 10 years of progress. Arch Dis Child 2011;96:998-1002.

8. Sovtic A, Minic P, Vukcevic M, et al. Home mechanical ventilation in children is feasible in developing countries. Pediatr Int 2012;54:676-81.

9. González Cortés R, Bustinza Arriortua A, Pons Ódena M, et al. Domiciliary mechanical ventilation in children: a Spanish multicentre study. An Pediatr (Barc) 2013;78:227-33.

10. McDougall CM, Adderley RJ, Wensley DF, et al. Long-term ventilation in children: longitudinal trends and outcomes. Arch Dis Child 2013;98:660-5.

11. Amin R, Sayal P, Syed F, et al. Pediatric long-term home mechanical ventilation: twenty years of follow-up from one Canadian center. Pediatr Pulmonol 2014;49:816-24.

12. Appierto L, Cori M, Bianchi R, et al. Home care for chronic respiratory failure in children: 15 years experience. Paediatr Anaesth 2002;12:345-50. 
13. Teague WG. Non-invasive positive pressure ventilation: current status in paediatric patients. Paediatr Respir Rev 2005;6:52-60.

14. Pavone M, Verrillo E, Caldarelli V, et al. Non-invasive positive pressure ventilation in children. Early Hum Dev 2013;89(Suppl 3):S25-31.

15. Fauroux B, Louis $B$, Hart N, et al. The effect of back-up rate during non-invasive ventilation in young patients with cystic fibrosis. Intensive Care Med 2004;30:673-81.

16. Fauroux B, Nicot F, Essouri S, et al. Setting of noninvasive pressure support in young patients with cystic fibrosis. Eur Respir $J$ 2004;24:624-30.

17. Fauroux B. Why, when and how to propose noninvasive ventilation in cystic fibrosis? Minerva Anestesiol 2011;77:1108-14.

18. Flight WG, Shaw J, Johnson S, et al. Long-term non-invasive ventilation in cystic fibrosis-Experience over two decades. J Cyst Fibrosis 2012;11:187-92.

19. Guilleminault C, Philip P, Robinson A. Sleep and neuromuscular disease: bilevel positive airway pressure by nasal mask as a treatment for sleep disordered breathing in patients with neuromuscular disease. J Neurol Neurosurg Psychiatry 1998;65:225-32.

20. Baydur A, Layne $\mathrm{E}$, Aral $\mathrm{H}$, et al. Long term non-invasive ventilation in the community for patients with musculoskeletal disorders: 46 year experience and review. Thorax 2000;55:4-11.

21. Mellies U, Dohna-Schwake C, Stehling F, et al. Sleep disordered breathing in spinal muscular atrophy. Neuromuscular Disord 2004; 14:797-803

22. Suresh S, Wales P, Dakin C, et al. Sleep-related breathing disorder in Duchenne muscular dystrophy: disease spectrum in the paediatric population. J Paediatr Child Health 2005;41:500-3.

23. Ward S, Chatwin M, Heather S, et al. Randomised controlled trial of non-invasive ventilation (NIV) for nocturnal hypoventilation in neuromuscular and chest wall disease patients with daytime normocapnia. Thorax 2005;60:1019-24.

24. Simonds AK. Recent advances in respiratory care for neuromuscular disease. Chest 2006;130:1879-86.

25. Waters KA, Everett $F$, Sillence $D$, et al. Breathing abnormalities in sleep in achondroplasia. Arch Dis Child 1993;69:191-6.

26. Waters KA, Everett FM, Bruderer JW, et al. Obstructive sleep apnea: the use of nasal CPAP in 80 children. Am J Respir Crit Care Med 1995;152:780-5.

27. Marcus CL, Ward SL, Mallory GB, et al. Use of nasal continuous positive airway pressure as treatment of childhood obstructive sleep apnea. J Pediatr 1995;127:88-94.

28. McNamara F, Sullivan CE. Obstructive sleep apnea in infants and its management with nasal continuous positive airway pressure. Chest 1999;116:10-16.

29. Kirk VG, Morielli A, Gozal D, et al. Treatment of sleep-disordered breathing in children with myelomeningocele. Pediatr Pulmonol 2000;30:445-52.

30. Murray C, Seton C, Prelog K, et al. Arnold Chiari type 1 malformation presenting with sleep disordered breathing in well children. Arch Dis Child 2006;91:342-3.

31. Weese-Mayer DE, Berry-Kravis EM, Ceccherini I, et al. ATS clinical policy statement: congenital central hypoventilation syndrome: genetic basis, diagnosis and management. Rev Mal Respir 2013;30:706-33.

32. Winfield NR, Barker NJ, Turner ER, et al. Non-pharmaceutical management of respiratory morbidity in children with severe global developmental delay. Cochrane Database Syst Rev 2014;10: CD010382.

33. Sullivan CE, McNamara F, Waters KA, et al. Nasal CPAP: use in the management of infantile apnea. Sleep 1993;16(8 Suppl):S108-11; discussion $\mathrm{S} 111-3$

34. Downey R III, Perkin RM, MacQuarrie J. Nasal continuous positive airway pressure use in children with obstructive sleep apnea younger than 2 years of age. Chest 2000;117:1608-12.

35. Essouri S, Nicot $F$, Clément $A$, et al. Noninvasive positive pressure ventilation in infants with upper airway obstruction: comparison of continuous and bilevel positive pressure. Intensive Care Med 2005:31:574-80.

36. Markström A, Sundell K, Stenberg N, et al. Long-term non-invasive positive airway pressure ventilation in infants. Acta Paediatr 2008;97:1658-62.

37. Khirani S, Ramirez A, Aloui S, et al. Continuous positive airway pressure titration in infants with severe upper airway obstruction or bronchopulmonary dysplasia. Crit Care 2013;17:R167.

38. Choo-Kang LR, Ogunlesi FO, McGrath-Morrow SA, et al. Recurrent pneumothoraces associated with nocturnal noninvasive ventilation in a patient with muscular dystrophy. Pediatr Pulmonol 2002;34:73-8.

39. Katz SL, Gaboury I, Keilty K, et al. Nocturnal hypoventilation: predictors and outcomes in childhood progressive neuromuscular disease. Arch Dis Child 2010;95:998-1003.
40. Liner LH, Marcus CL. Ventilatory management of sleep-disordered breathing in children. Curr Opin Pediatr 2006;18:272-6.

41. Koontz KL, Slifer KJ, Cataldo MD, et al. Improving pediatric compliance with positive airway pressure therapy: the impact of behavioral intervention. Sleep 2003;26:1010-15.

42. Marcus CL, Rosen G, Ward SL, et al. Adherence to and effectiveness of positive airway pressure therapy in children with obstructive sleep apnea. Pediatrics 2006;117:e442-51.

43. O'Donnell AR, Bjornson CL, Bohn SG, et al. Compliance rates in children using noninvasive continuous positive airway pressure. Sleep 2006;29:651-8.

44. Marcus CL, Beck SE, Traylor J, et al. Randomized, double-blind clinical trial of two different modes of positive airway pressure therapy on adherence and efficacy in children. J Clin Sleep Med 2012;8:37-42.

45. Prashad PS, Marcus CL, Maggs J, et al. Investigating reasons for CPAP adherence in adolescents: a qualitative approach. J Clin Sleep Med 2013;9:1303-13.

46. Ramirez A, Khirani S, Aloui S, et al. Continuous positive airway pressure and noninvasive ventilation adherence in children. Sleep Med 2013;14:1290-4

47. Dohna-Schwake C, Podlewski P, Mellies U. Non-invasive ventilation reduces respiratory tract infections in children with neuromuscular disorders. Pediatr Pulmonol 2008;43:67-71.

48. Toussaint M, Soudon P, Kinnear W. Effect of non-invasive ventilation on respiratory muscle loading and endurance in patients with Duchenne muscular dystrophy. Thorax 2008;63:430-4.

49. Elliott MW, Simonds AK, Carroll MP, et al. Domiciliary nocturnal nasal intermittent positive pressure ventilation in hypercapnic respiratory failure due to chronic obstructive lung disease: Effects on sleep and quality of life. Thorax 1992;47:342-8.

50. McNamara F, Sullivan CE. Treatment of obstructive sleep apnea syndrome in children. Sleep 2000;23(SUPPL. 4):S142-6.

51. Marcus CL, Radcliffe J, Konstantinopoulou S, et al. Effects of positive airway pressure therapy on neurobehavioral outcomes in children with obstructive sleep apnea. Am J Respir Crit Care Med 2012;185:998-1003.

52. Simonds AK, Elliott MW. Outcome of domiciliary nasal intermittent positive pressure ventilation in restrictive and obstructive disorders. Thorax 1995;50:604-9.

53. Leger P, Bedicam JM, Cornette A, et al. Nasal intermittent positive pressure ventilation: Long-term follow-up in patients with severe chronic respiratory insufficiency. Chest 1994;105:100-5.

54. Edwards EA, Nixon GM. Paediatric home ventilatory support: changing milieu, proactive solutions. J Paediatr Child Health 2013;49:13-18.

55. Annane D, Orlikowski D, Chevret S, et al. Nocturnal mechanical ventilation for chronic hypoventilation in patients with neuromuscular and chest wall disorders. Cochrane Database Syst Rev 2007;(4): CD001941. http://onlinelibrary.wiley.com/doi/10.1002/14651858. CD001941.pub2/abstract

56. Kuhle S, Urschitz MS, Eitner S, et al. Interventions for obstructive sleep apnea in children: a systematic review. Sleep Med Rev 2009;13:123-31.

57. Teoh L, Hurwitz M, Acworth JP, et al. Treatment of obstructive sleep apnoea for chronic cough in children. Cochrane Database Syst Rev 2011(4):CD008182.

58. Goyal V, Masters IB, Chang AB. Interventions for primary (intrinsic) tracheomalacia in children. Cochrane Database Syst Rev 2012;10: CD005304.

59. Marcus CL, Brooks LJ, Draper KA, et al. Diagnosis and management of childhood obstructive sleep apnea syndrome. Pediatrics 2012;130:e714-55.

60. Afsharpaiman S, Saburi A, Waters KA. Respiratory difficulties and breathing disorders in achondroplasia. Paediatr Respir Rev 2013;14:250-5

61. Venekamp RP, Hearne BJ, Chandrasekharan D, et al. Tonsillectomy or adenotonsillectomy versus non-surgical management for sleep-disordered breathing in children. Cochrane Database Syst Rev 2014;(6). http://onlinelibrary.wiley.com/doi/10.1002/14651858. CD011165/abstract

62. Bragge $\mathrm{P}$, Clavisi $\mathrm{O}$, Turner $\mathrm{T}$, et al. The global evidence mapping initiative: scoping research in broad topic areas. BMC Med Res Methodol 2011;11:92

63. Arksey H, O'Malley L. Scoping studies: Towards a methodological framework. Int J Soc Res Methodol 2005;8:19-32.

64. Shamseer L, Moher D, Clarke M, et al. Preferred reporting items for systematic review and meta-analysis protocols (prisma-p) 2015: elaboration and explanation. BMJ 2015;349:g7647.

65. Boluyt N, Tjosvold L, Lefebvre C, et al. Usefulness of systematic review search strategies in finding child health systematic reviews in MEDLINE. Arch Pediatr Adolesc Med 2008;162:111-16. 